\title{
Acute Stroke with Concomitant Acute Myocardial Infarction: Will You Thrombolyse?
}

\author{
Rita Maciel, ${ }^{\mathrm{a}, \mathrm{b}}$ Raquel Palma, ${ }^{\mathrm{b}, \mathrm{c}}$ Pedro Sousa, ${ }^{\mathrm{d}}$ Fátima Ferreira, ${ }^{\mathrm{b}, \mathrm{c}}$ Hipólito Nzwalo ${ }^{\mathrm{b}, \mathrm{c}, \mathrm{e}}$ \\ ${ }^{a}$ Department of Internal Medicine, Centro Hospitalar do Algarve (CHA), Faro, Portugal \\ ${ }^{\mathrm{b}}$ Stroke Unit, Centro Hospitalar do Algarve (CHA), Faro, Portugal \\ 'Department of Neurology, Centro Hospitalar do Algarve (CHA), Faro, Portugal \\ ${ }^{\mathrm{d} D}$ Department of Cardiology, Centro Hospitalar do Algarve (CHA), Faro, Portugal \\ eDepartment of Biomedical Sciences and Medicine, University of Algarve, Faro, Portugal
}

\section{Dear Sir:}

Concomitant occurrence of acute myocardial infarction (MI) and stroke is infrequently encountered in emergent patients. Acute MI within the previous 3 months is considered a relative contraindication for therapy with alteplase or intravenous tissue plasminogen activator (IV rtPA). ${ }^{1}$ The use of IV rtPA for stroke in patients with a recent $\mathrm{MI}$ is associated with an increased risk of cardiac rupture, secondary to breakdown of the existing fibrin clot within the necrotic myocardium and/or degradation of collagen ${ }^{2}$. Whether it is appropriate to perform thrombolysis in an emergent patient with concomitant ischemic stroke and MI remains a matter for debate.

A 44-year-old Caucasian man with a past history of binge drinking and heavy smoking since childhood, presented to the emergency department (ED) with a history of sudden onset of focal neurologic deficits, which had stared 1 hour previously. Neurological examination revealed dysarthria, left-sided homonymous hemianopia, facial central paresis, hemiparesis, and hemineglect (National Institutes of Health Stroke Scale [NIHSS] score, 11). His general examination was unremarkable. Blood pressure was $110 / 70 \mathrm{mmHg}$, temperature was $36.8^{\circ} \mathrm{C}$, and pulse was $61 \mathrm{bpm}$. There was no jugular venous distension. He also complained of an intermittent vague ache in the anterior thoracic region and left shoulder, which had been present for the preceding 48 hours. The patient's electrocardiogram showed signs compatible with inferior MI with 2:1 atrioventricular block (Figure 1A). Cardiac troponin I level was elevated at $16.045 \mathrm{ng} / \mathrm{mL}$ (normal value, $\leq 0.04 \mathrm{ng}$ /
$\mathrm{mL})$. Emergent bedside transthoracic echocardiography showed the presence of inferior wall hypokinesis with good left ventricular systolic function. Computed tomography brain scan (brain CT) showed early signs of ischemia in the territory of the right middle cerebral artery (Figure 1B).

IV t-PA $(0.9 \mathrm{mg} / \mathrm{kg}$ over 1 hour, total dose $80 \mathrm{mg})$ was administered $2 \mathrm{~h}$ after stroke onset. The patient's condition improved with thrombolysis (NIHSS score, 4). Approximately 10 hours after thrombolysis, he developed persistent bradycardia (40-50 bpm) and hypotension (100-90 $\mathrm{mmHg}$ systolic, $40-60 \mathrm{mmHg}$ diastolic), requiring treatment with volume expanders. Transthoracic echocardiography revealed expansion of the MI to the right ventricle, without cardiac tamponade or depression of systolic function. Brain CT was repeated after 24 hours and showed the presence of ischemia in the cortical territory of the right middle cerebral artery (Figure 1C). Results of the remaining investigations, including a metabolic panel (glucose levels, hemogram, blood coagulation times, ionogram, renal function, hepatic enzymes), were normal. The patient was transferred to the intensive care unit after successful resuscitation for ventricular fibrillation. He remained in the intensive care unit for 2 consecutive weeks, during which time he had several medical complications, including refractory malignant arrhythmias with a recurrent need for resuscitation. Brain CT was repeated 8 days after the stroke and showed 2 new lesions in the posterior cerebral artery territory (Figure 1D). On discharge from the ICU, he had Psychomotor slowing, left-sided hemiparesis/hemihypesthesia and mild appendicular ataxia (NIHSS score, 9). Six months after the stroke, 

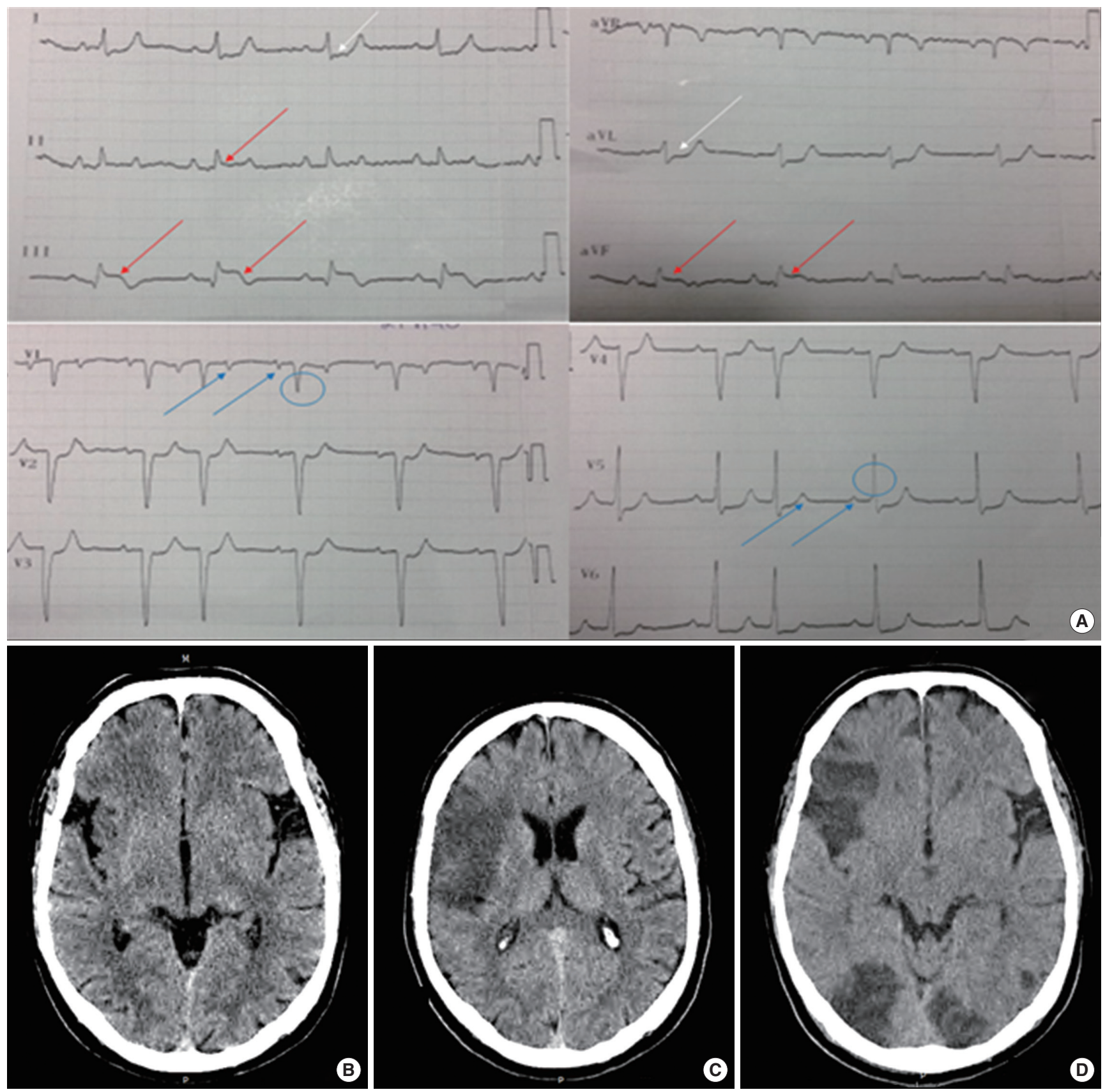

Figure 1. (A) Electrocardiogram showing sinus rhythm, with 2:1 conduction block; two p waves (blue arrows) and one QRS wave (blue circle) are shown. ST elevation is visible in leads II, III, and aVF (red arrows), with ST depression in leads I and aVL (white arrows). (B) Brain CT showing early signs of acute stroke (insular ribbon sign and hemispheric sulcus effacement) in the right MCA territory. (C) Established stroke in the cortical territory of the right MCA. (D) Additional bilateral ischemic stroke in the posterior territory.

he resumed his normal life (Rankin 2).

The inclusion of $\mathrm{MI}$ as a relative or absolute contraindication for IV rtPA in acute stroke is not evidence-based. ${ }^{1,2}$ Although cardiac wall rupture and tamponade are very rare in patients presenting acute stroke and MI, these complications may constitute a major barrier for IV rtPA in such patients. ${ }^{2}$ Factors known to be associated with an increased risk of cardiac wall rupture and tamponade include older age, female sex, large anterior MI, longer time from symptom onset to thrombolysis, infarct size, transmural extent, and pericardial involvement. ${ }^{3,4}$ The overall frequency of these complications is approximately $1 \%$ in patients treated with thrombolysis for acute MI. ${ }^{3}$ However, in comparison to controls, with the exception of patients older than 75 years, the occurrence of and the risk of mortality from cardiac rupture is not increased after treatment of acute MI with rtPA. ${ }^{3-5}$

There are no evidence-based guidelines for the management 
of patients with concomitant acute MI and acute stroke, nor are there published clinical studies addressing the decision-making process in such cases. Depending on the clinical picture, physicians may focus on the management of either the stroke or the MI. Obviously, independent management of one thrombosed territory will delay the treatment of the other vascular bed. Although superior to thrombolysis for acute $\mathrm{MI}^{6}{ }^{6}$ primary percutaneous coronary intervention would delay rtPA for stroke, increasing the risk of severe neurological disability in our case. Furthermore, intravenous heparin bolus during, and dual antiplatelet therapy (aspirin plus clopidogrel) following coronary angioplasty, would potentiate the risk of hemorrhagic complications related to rtPA for stroke. Mechanical thrombectomy for acute stroke, a procedure that is not available in most hospitals including ours, could theoretically be combined with percutaneous coronary intervention. While we did not find any single report of such combined intervention, some authors believe that in the presence of acute ischemic emergent conditions affecting different territories or organs, intravenous thrombolytic therapy is a reasonable option. ${ }^{7}$

In a patient without demographic risk factors for cardiac tamponade, we decided to offer thrombolysis in a balanced attempt to treat both myocardial and brain infarction, after excluding any relevant change on bedside transthoracic echocardiography.

In conclusion, given the current knowledge limitations, treatment decisions in cases of concomitant acute MI and acute ischemic stroke should be individualized. The delicate balance between the presence or absence of risk factors for cardiac tamponade and the potential cardiac or neurological disability may guide the clinician in such a difficult scenario. Alteplase should probably not be avoided in eligible patients with ischemic stroke and $\mathrm{MI}$ in the absence of the aforementioned risk factors for cardiac complications associated with intravenous thrombolysis.

\section{References}

1. Jauch EC, Saver JL, Adams HP, Demaerschalk BM, Khatri P,
McMullen P et al. Guidelines for the early management of patients with acute ischemic stroke: a guideline for healthcare professionals from the American Heart Association/American Stroke Association. Stroke 2013;44:870-947.

2. De Silva DA, Manzano JJ, Chang HM, Wong MC. Reconsidering recent myocardial infarction as a contraindication for IV stroke thrombolysis. Neurology 2011;76:1838-1840.

3. Patel MR, Meine TJ, Lindblad L, Griffin J, Granger CB, Becker $\mathrm{RC}$ et al. Cardiac tamponade in the fibrinolytic era: analysis of $>100,000$ patients with ST-segment elevation myocardial infarction. Am Heart J 2006; 151:316-322.

4. Becker RC1, Hochman JS, Cannon CP, Spencer FA, Ball SP, Rizzo MJ et al. Fatal cardiac rupture among patients treated with thrombolytic agents and adjunctive thrombin antagonists: observations from the Thrombolysis and Thrombin Inhibition in Myocardial Infarction 9 Study. J Am Coll Cardiol 1999;33: 479-487.

5. Bueno H, Martínez-Sellés M, Pérez-David E, López-Palop R. Effect of thrombolytic therapy on the risk of cardiac rupture and mortality in older patients with first acute myocardial infarction. Eur Heart J 2005;26:1705-1711.

6. Keeley EC, Boura JA, Grines CL. Primary angioplasty versus intravenous thrombolytic therapy for acute myocardial infarction: a quantitative review of 23 randomised trials. Lancet 2003; 361:13-20.

7. Omar HR, Mangar D, Camporesi EM. Simultaneous thrombosis of 2 vascular territories: is thrombolytic therapy a better option? Am J Emerg Med 2013;31:1412-1413.

Correspondence: Hipólito Nzwalo

Hospital de Faro, Department of Neurology, Rua Leao Penedo, 8000-386 Faro, Portugal

Tel: +351968659413, Fax: +289.891.144

E-mail: nzwalo@gmail.com

Received: August 30, 2014

Revised: November 12, 2014

Accepted: November 17, 2014

The authors have no financial conflicts of interest. 